\title{
Development of Analytical Methods Utilizing Selectivity of Fluorous Affinity and Their Applications
}

\author{
Tadashi HAYAMA* \\ Faculty of Pharmaceutical Sciences, Fukuoka University, 8-19-1 Nanakuma, Johnan-ku, Fukuoka 814-0180, Japan
}

\begin{abstract}
Perfluoroalkyl-containing compounds show a unique "fluorous" property, which is related to the specific affinity, i.e. fluorophilicity, between perfluoroalkyl groups. Therefore, fluorous compounds can be easily and selectively isolated from non-fluorous compounds utilizing liquid-liquid extraction, solid phase extraction, and liquid chromatographic techniques via their fluorophilicity. We have also developed novel methods for the selective analysis of biogenic-related compounds using fluorous separation techniques. These methods involve the attachment of perfluoroalkyl groups to target analytes, followed by their selective separation from endogenous biological matrices with appropriate fluorous separation techniques. Because the perfluoroalkylated compounds are generally not included in biological matrices, highly selective analysis of perfluoroalkyl-attached target analytes is possible. This review summarizes our recent works concerning biogenic-related compound analysis methods using fluorous separation techniques.
\end{abstract}

Keywords: Fluorous; Perfluoroalkyl; Biogenic-related compounds; Selective analysis

\section{Introduction}

Highly sensitive and selective analysis methods are necessary for accurate and precise identification and quantification of analytes of interest in complex biological samples. Although the sensitivity for analytes has improved remarkably in recent years with improvements in the performance of analytical instruments, improving the selectivity for analytes is more challenging because the isolation of target analytes from biological matrices is generally difficult. Therefore, a novel method for improving selectivity rather than sensitivity is required for the analysis of biological samples.

Perfluoroalkyl groups have many interesting characteristics, such as high electronegativity, low polarizability, and good thermal and chemical stabilities. Furthermore, they have a remarkable affinity for one another, which is termed "fluorophilicity", in spite of their lipophobicity and hydrophobicity. Utilizing their fluorous affinities, perfluoroalkyl-containing (fluorous) compounds can be easily distinguished from non-fluorous compounds by using their solvent (e.g., perfluoroalkanes) or material (e.g., perfluoroalkyl-modified silica gel) analogues [1-4]. Although fluorous separation techniques, such as fluorous biphasic liquid-liquid extraction (F-LLE) and fluorous solid phase extraction (F-SPE), have been conventionally used for enrichment and/or purification in organic biomolecule synthesis, their application to biogenic-related compound analyses has been recently reported $[5,6]$. We also developed novel liquid chromatography (LC) methods for the selective analysis of target analytes in complex biological samples. The concept of these methods is the derivatization of target biogenic molecules with fluorous reagents and selective retention and separation of derivatives with a perfluoroalkyl-modified stationary phase (fluorous) LC column via fluorophilicity, not hydrophobicity. Because the non-derivatized (non-fluorous) endogenous components of a biological matrix cannot be retained on the column, the highly selective and accurate analysis of fluorous derivatives can be performed without interference from biological matrix. In addition, to demonstrate the wide applicability of fluorous technologies, novel pretreatment methods of

Received: 7 December 2015

Accepted: 19 December 2015

J-STAGE Advance Published: 26 December 2015

DOI: 10.15583/jpchrom.2015.039 
biological samples with F-LLE and F-SPE were developed for conventional LC or tandem mass spectrometry (MS) analysis.

The objective of this review is to describe our recent work utilizing fluorous separation techniques for the selective analysis of biomolecules in complex biological sample matrices.

2. Selective analysis of native fluorescent biogenic amines using the fluorous derivatization method

Target analytes can easily be made "fluorous" by the attachment of perfluoroalkyl groups with fluorous reagents, which have ethyl or propyl spacers between the reactive functional groups and the perfluoroalkyl groups to minimize fluorine electronic effects. Native fluorescent biogenic amines, such as 3,4-dihydroxy-L-phenylalanine (DOPA), dopamine (DA), norepinephrine (NE), epinephrine (E), metanephrine (MN), tryptophan (Trp), 5-hydroxytryptophan (5-HTrp), and serotonin (5-HT), were derivatized with a 2-(perfluorooctyl)ethyl isocyanate (PFOEI) fluorous reagent (Fig. 1) [7]. The derivatization reaction proceeded at $60^{\circ} \mathrm{C}$ for $10 \mathrm{~min}$, and the reaction mixture was directly injected into the LC system without any pretreatment after the addition of aqueous ammonia to consume the excess reagent.

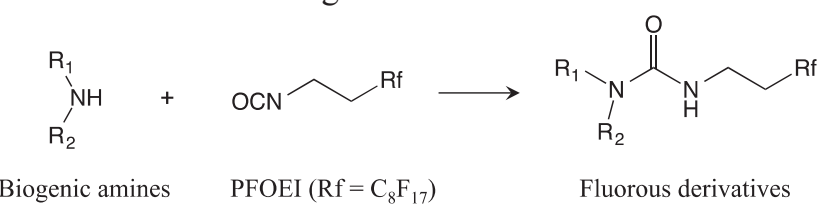

Fig. 1. Fluorous derivatization of biogenic amines with PFOEI.

The obtained derivatives could be separated within 30 min after selective retention on a perfluoroalkyl-modified stationary phase (fluorous) LC column, and detected by their native fluorescent characteristics (Fig. 2A). The relationship between the concentrations of examined biogenic amines and the peak heights were linear over the concentration range of $1-100 \mathrm{nmol} / \mathrm{mL}$ for DOPA, $0.5-25$ $\mathrm{nmol} / \mathrm{mL}$ for $\mathrm{DA}, \mathrm{NE}$, and $\mathrm{MN}$, and $0.1-10 \mathrm{nmol} / \mathrm{mL}$ for $\mathrm{E}$, Trp, 5-HTrp, and 5-HT. The correlation coefficients of the calibration curves were greater than 0.9989 . The relative standard deviations (RSDs) of the intra-day precision values obtained by six replicate analyses of standard solutions were within $3.2 \%$. The limits of detection [LODs, signal-to-noise ratio $(S / N)$ of 3] were less than $640 \mathrm{fmol}$ on column for all examined biogenic amines.

The possibility of applying this fluorous derivatization method to the effective separation of a biological matrix on the fluorous LC column was evaluated using a human urine sample. After deproteinization with tetrahydrofuran, the human urine sample was derivatized with PFOEI, followed by fluorous LC analysis. The typical chromatogram obtained from the derivatized human urine sample is depicted in Fig. 2B. As expected, the underivatized urine components were eluted earlier from the fluorous LC column (within $15 \mathrm{~min}$ ), and they were completely distinguished from the examined biogenic amine derivatives. The determination results (mean $\pm \mathrm{SD}$ ) of the examined biogenic amines in human urine from healthy subjects $(n=7)$ were as follows: DOPA $5.4 \pm 2.4$, DA $5.0 \pm$ 1.6 , NE $4.4 \pm 1.6$, E $1.9 \pm 1.1$, MN $3.6 \pm 1.0, \operatorname{Trp} 32 \pm 6.5$, 5 -HTrp $1.4 \pm 0.69$, and 5-HT $2.6 \pm 0.81 \mathrm{nmol} / \mathrm{mL}$ urine. These results agree well with previously reported data [8-10].
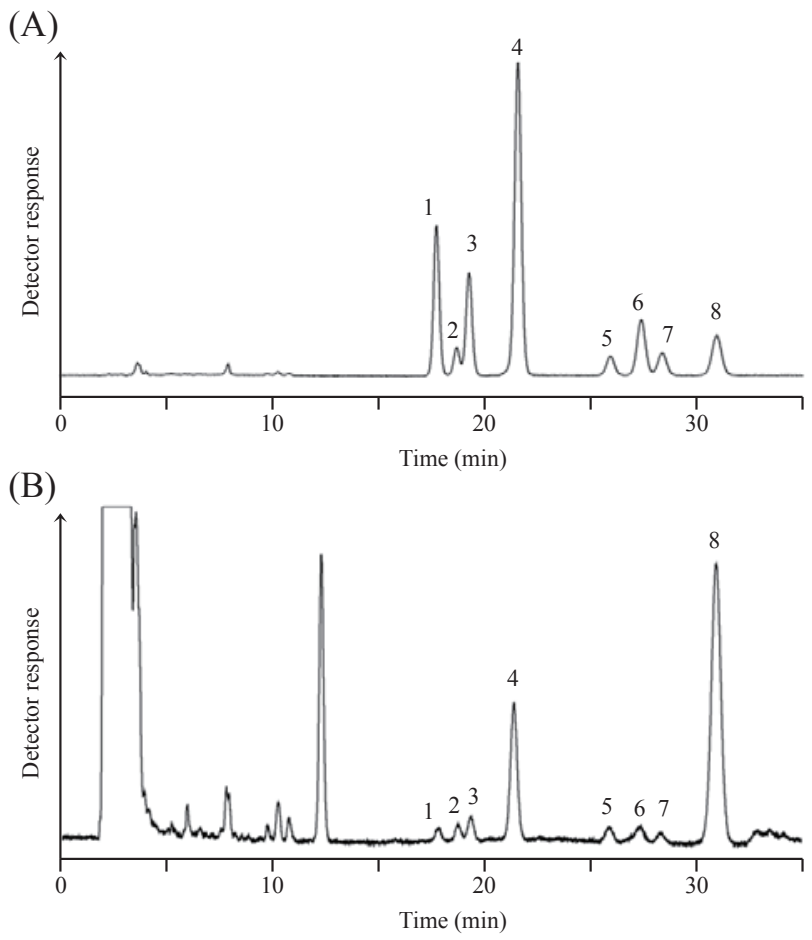

Fig. 2. Chromatograms obtained from (A) standard solution (DOPA: 50 pmol on column; others: 5 pmol on column) and (B) healthy human urine sample with PFOEI derivatization using fluorous LC separation. Peaks: 1, DOPA; 2, NE, 3, 5-HTrp; 4, E; 5, DA; 6, 5-HT; 7, MN; 8, Trp. LC conditions: column, Fluofix-II $120 \mathrm{E}$ column $(250 \times 4.6 \mathrm{~mm}$ ID, Wako $)$; mobile phase, acetonitrile - water - trifluoroacetic acid (60:40:0.05, v/v); flow rate, 1.0 $\mathrm{mL} / \mathrm{min}$; column temperature, $30^{\circ} \mathrm{C}$; fluorescence detection, ex 280 $\mathrm{nm}$ and em $320 \mathrm{~nm}$. Reprinted with permission from Ref. [7].

\section{Selective analysis of biogenic primary amines using LC-MS/MS with the fluorous derivatization method}

The fluorous derivatization method has also been very useful for complex biological sample analysis with LC-tandem mass spectrometry (MS/MS), because this method has enabled elimination of matrix-induced suppression effects during analysis. It is thought that the matrix effects are caused by ionization competition between the analytes and co-eluting components of the biological matrix in the electrospray ionization source $[11,12]$. Many 
researchers have used stable isotope-labeled internal standards to overcome matrix effects, although the use of such internal standards, especially for deuterized ones, is sometimes limited because of unexpected behavior, such as different recoveries or retention times, during pretreatment or LC separation [13,14]. On the other hand, our developed separation-oriented derivatization method using fluorous affinity is useful for eliminating matrix effects because non-fluorous matrix components are formerly eluted and clearly separated from the fluorous derivatives by fluorous LC $[15,16]$. We applied this method to the accurate analysis of trace amounts of biogenic primary amines [DA, NE, 3-methoxytyramine (3-MT), normetanephrine (NM), tyramine (Tyr), 5-HT, tryptamine (Tryp), 5-methoxytryptamine (5-MT), and histamine (Hist)] in human plasma samples [17]. Although biogenic amines could be derivatized to their perfluoroalkylated forms with PFOEI as mentioned above, derivatization with a fluorous aldehyde reagent, $2 \mathrm{H}, 2 \mathrm{H}, 3 \mathrm{H}, 3 \mathrm{H}$-perfluoroundecan-1-al (PFUA), enabled more sensitive, selective, and accurate analysis by LC-MS/MS. The primary amino groups of the target amines were easily transformed to binary fluorous derivatives with PFUA in the presence of the reductant (2-picoline borane) via a reductive amination reaction [18,19] (Fig. 3).

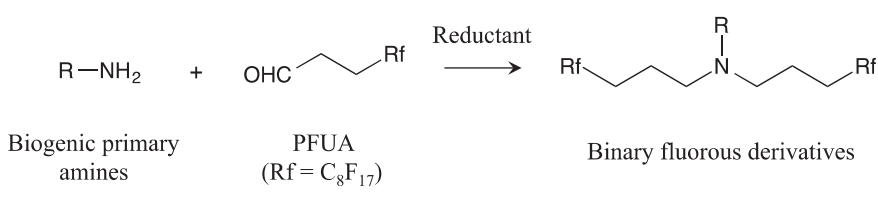

Fig. 3. Fluorous derivatization of biogenic primary amines with PFUA via reductive amination.

The resulting binary fluorous derivatives of the examined amines were strongly retained on the fluorous LC column due to their strong fluorophilicity. However, the derivatives could not be eluted from the column with a weakly acidic mixture of water, methanol, and acetonitrile as the mobile phase. This problem was solved by the use of a polar fluorous alcohol, such as 2,2,2-trifluoroethanol (TFE), as a co-eluent in the mobile phase, providing good retention times and sharp peak shapes for the derivatives. Fluorous solvents are typically very nonpolar and usually cannot be used in reversed-phase LC. However, because TFE can be miscible with many organic solvents, it has been utilized as a controllable solvent for the elution of fluorinated compounds in fluorous and reversed-phase LC [20,21]. Furthermore, we screened the retention behavior of the derivatives on several commercially available fluorous LC columns: Fluorous LC column are commercially available: examples include Fluophase RP (Thermo Fisher Scientific, San Jose, CA, USA), Fluofix-II 120E (Wako Pure Chemicals, Osaka, Japan), and

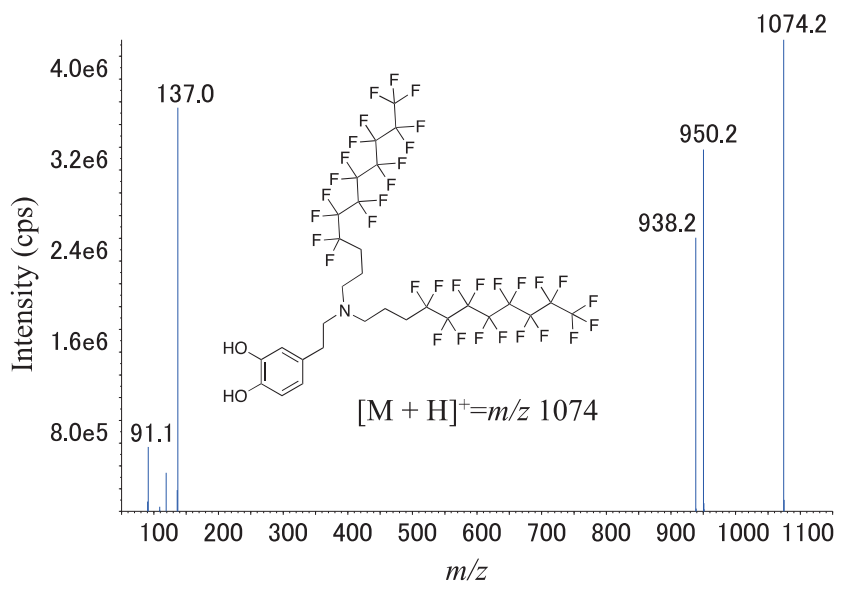

Fig. 4. ESI-MS/MS spectrum of the binary fluorous-derivatized DA (precursor ion: $m / z$ 1074). The collision-induced dissociation energy was $60 \mathrm{eV}$. Reprinted with permission from Ref. [17].

FluoroSep-RP Propyl (ES industry, West Berlin, NJ, USA), and FluoroSep-RP Octyl (ES industry) columns. Among them, better shapes and sharpness of the binary fluorous derivative peaks were obtained using a FluoroSep-RP Propyl column.

The quantification of derivatives was performed by multiple reaction monitoring (MRM) mode in MS/MS. The protonated ions $\left([\mathrm{M}+\mathrm{H}]^{+}\right)$were obtained by positive ionization mode in the first quadrupole for all derivatives. Furthermore, when the fragment ions were scanned in the third quadrupole, common fragment ions $(\mathrm{m} / \mathrm{z} 950$ and/or 938 ) and some characteristic ions corresponding to cleavage ions of the form $\left[\mathrm{M}+\mathrm{H}-\mathrm{NH}\left(\mathrm{C}_{3} \mathrm{H}_{6} \mathrm{C}_{8} \mathrm{~F}_{17}\right)_{2}\right]^{+}$, e.g. $\mathrm{m} / z 137$ for DA (Fig. 4), were observed for all derivatives except for $\mathrm{NE}$ and NM. In the case of $\mathrm{NE}$ and $\mathrm{NM}$, the $[\mathrm{M}+\mathrm{H}-$ $\left.\mathrm{H}_{2} \mathrm{O}\right]^{+}$were obtained as the dominant fragment ions. The results demonstrated that the examined biogenic primary amines were transformed to binary fluorous derivatives. For the most sensitive quantitative analysis of the examined biogenic primary amines, the most intense precursor-product transitions were set in the MRM mode. Under the derivatization and LC-MS/MS analysis conditions, the relationship between the peak area of the derivatives and the concentrations of the examined biogenic primary amines were linear over a concentration range of 0.01-1 nM. The correlation coefficients of the calibration curves were greater than 0.9978. The RSDs of the inter-day precision values obtained by repetitive analysis ( $n$ $=6$ ) of the standard solutions were within $7.4 \%$. The LODs $(S / N=3)$ were $7.8-26$ amol on column.

To evaluate the feasibility of eliminating matrix effects, the method was validated using human plasma samples. According to the report by Matuszewski et al. [12], the matrix effect values were calculated by the response from derivatized standard amines (non-matrix) and spiked human plasma samples (matrix). The results are shown in Table 1. The values were in the range of $95.9-106 \%$ for all 
Table 1. Evaluation of matrix effects.

\begin{tabular}{lccc}
\hline & \multicolumn{3}{c}{ Matrix effect $($ mean $\pm \mathrm{SD} \%, n=3)$} \\
\cline { 2 - 4 } & $0.01 \mathrm{nM}$ & $0.1 \mathrm{nM}$ & $1 \mathrm{nM}$ \\
\hline DA & $101 \pm 5.4$ & $102 \pm 8.2$ & $97.5 \pm 5.1$ \\
NE & $100 \pm 6.6$ & $99.4 \pm 4.9$ & $98.3 \pm 4.2$ \\
3-MT & $100 \pm 3.6$ & $99.2 \pm 6.7$ & $101 \pm 1.6$ \\
NM & $100 \pm 2.3$ & $106 \pm 2.3$ & $97.2 \pm 6.7$ \\
Tyr & $103 \pm 1.1$ & $100 \pm 4.4$ & $100 \pm 4.4$ \\
5-HT & $97.1 \pm 3.8$ & $102 \pm 1.2$ & $102 \pm 1.2$ \\
Tryp & $96.5 \pm 0.9$ & $103 \pm 6.1$ & $103 \pm 6.1$ \\
5-MT & $100 \pm 3.9$ & $96.2 \pm 5.4$ & $96.2 \pm 5.4$ \\
Hist & $103 \pm 1.1$ & $103 \pm 8.2$ & $103 \pm 8.2$ \\
\hline
\end{tabular}

concentrations examined. Therefore, it was demonstrated that the fluorous derivatization method enables the accurate and precise determination of biogenic amines in biological samples with LC-MS/MS.

The determination results obtained from several native human plasma samples $(n=9)$ were as follows: DA 0.10-0.95, NE 0.10-1.1, 3-MT 0.02-0.50, NM 0.02-0.43, Tyr $0.42-4.2, \quad 5-\mathrm{HT} \quad 8.9-57$, Tryp 1.2-3.2, 5-MT < LOD-0.04, and Hist $0.79-28 \mathrm{pmol} / \mathrm{mL}$ plasma. These values are also in good agreement with previously reported data [22-24].

\section{Direct tandem MS analysis for amino acids using the fluorous derivatization and purification method}

The fluorous derivatization method was applied to direct tandem MS analysis of amino acids in plasma samples. Direct tandem MS methods have been developed for the screening of metabolic disorders by determining amino acid levels rapidly [25-27]. Although these methods have allowed effective and economic analysis of amino acids, direct injection of biological sample solutions without chromatographic column separation often causes inaccurate quantification results and shortening of the MS instrument lifetime. Therefore, to improve the direct tandem MS analysis method for amino acids, fluorous derivatization followed by purification with a perfluoroalkyl-modified monolithic silica-based solid phase extraction (monolithic F-SPE) by spin column was performed [28]. Although the SPE purification step post-derivatization may be laborious, the monolithic spin column enables rapid, simple, and reproducible sample extraction. Generally, commercially available fluorous particle-type silica columns are consisted of a $\mathrm{C}_{6} \mathrm{~F}_{13}$ or $\mathrm{C}_{8} \mathrm{~F}_{17}$ bonded silica-gel-based stationary phase [20]. Therefore, a fluorous spin column consisting of $\mathrm{C}_{6} \mathrm{~F}_{13}$ attached with an ethylene spacer to a silica-based monolithic stationary phase was used.

Amino acids (L-alanine, L-arginine, L-aspartic acid, L-glutamic acid, glycine, L-histidine, L-leucine, L-isoleucine, L-lysine, L-phenylalanine, L-serine, L-threonine, L-tyrosine, and L-valine) were derivatized to their binary fluorous forms using PFUA via a slightly modified version of the method described above for biogenic amine derivatization. After derivatization, an aliquot of the resulting reaction mixture was subjected to monolithic F-SPE spin column purification. Then, the obtained extracts were directly injected into the tandem MS system without column separation, and the derivatives were detected by MRM mode in positive ESI.

Utilizing the fluorous spin column, all of the examined amino acid derivatives were successfully extracted with good reproducibility, although the recovery of some, such as Asp, Glu, and Tyr, were relatively low. To demonstrate the selectivity of the fluorous spin column, a monolithic ODS spin column was used for extraction under the same conditions as a comparison (Fig. 5). As a result, almost all of the amino acid derivatives, except for L-lysine that was derivatized to the tetra-perfluoroalkylated form, could not be retained and passed through the monolithic ODS column. These results therefore confirmed that the monolithic F-SPE column enabled selective extraction of the fluorous derivatives via fluorophilicity, not hydrophobicity.

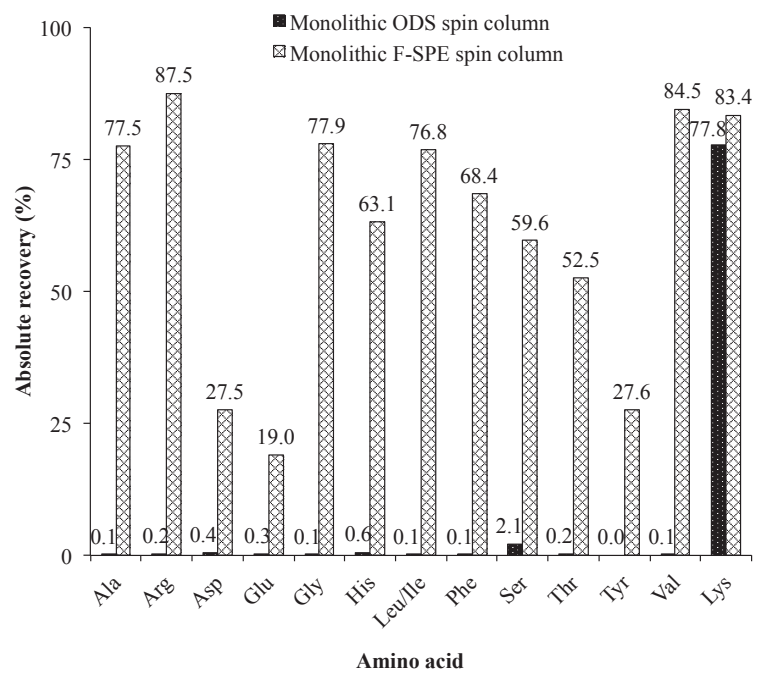

Fig. 5. Comparison of amino acid derivative recovery from the monolithic F-SPE spin column with that the monolithic ODS spin column. Reprinted with permission from Ref. [28].

A validation study was performed using non-spiked and spiked $(50,100$, and $500 \mathrm{nmol} / \mathrm{mL}$ plasma) human plasma samples. The determination results of amino acids in the non-spiked plasma sample obtained with this method agreed well with those of the literature, referring to the Human Metabolome Database (HMDB) [29]. The accuracy data obtained from the spiked plasma samples were in the range of $82.5-118 \%$ for all amino acids except for L-glutamic acid, for which the accuracy was relatively low (61.9-67.2\%). However, because the data was reproducible for all targeted amino acids, their quantitative analysis with the present method was sufficiently suitable. 

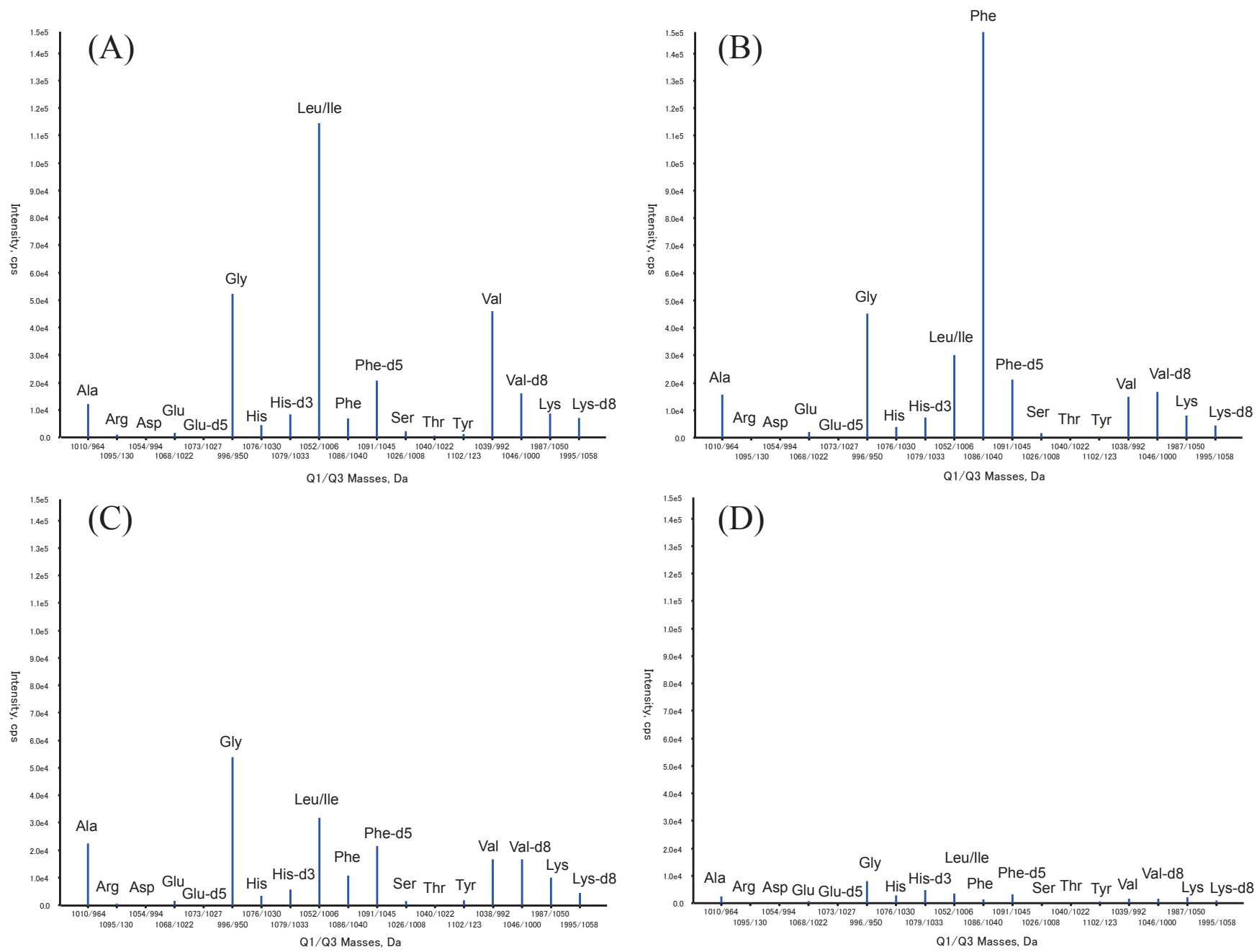

Fig. 6. MRM profiles obtained from (A) MSUD model and (B) PKU model, and control mouse plasma samples (C) with purification and (D) without purification by monolithic F-SPE spin column. Reprinted with permission from Ref. [28].

Furthermore, to confirm the applicability of this method to the diagnosis of metabolic abnormalities, plasma samples obtained from control mice and model mice with maple syrup urine disease (MSUD) and phenylketonuria (PKU) were analyzed (Fig. 6). It is known that MSUD and PKU lead to elevation of branched-chain amino acids (L-leucine, L-isoleucine, and L-valine) and L-phenylalanine levels, respectively, in blood. In the determination results obtained by this method, the corresponding amino acid levels of MSUD and PKU model mice were also significantly elevated compared to those of the control mice. On the other hand, suppression of peak intensity, thought to be caused by matrix effects, was observed from the MRM profiles in the analysis of control mice plasma samples without purification by monolithic F-SPE. From these results, it can be seen that this method enabled the determination of amino acids in plasma with the elimination of matrix effects in tandem MS analysis. It is therefore a beneficial tool for the diagnosis of amino acid metabolic abnormalities.

\section{Selective fluorous biphasic extraction for nucleotides without derivatization}

A novel method for the selective extraction of nucleotides (AMP, ADP, ATP, GMP, GDP, and GTP) utilizing the fluorous biphasic liquid extraction system was developed [30]. Although the derivatization reaction has generally been required in fluorous separation techniques, this method enabled the extraction of nucleotides into fluorous solvents without derivatization, utilizing 4,4,5,5,6,6,7,7,8,8,9,9,10,10,11,11,11-heptadecafluoroundecylamine (HFUA) as a fluorous ion-pair reagent (Fig. 7). The fluorous biphasic separation method has been often used to extract heavily fluorous compounds ( $>60 \%$ fluorine in the molecule). In contrast, lightly fluorous compounds, such as HFUA, cannot be extracted into fluorous solvents, such as perfluoroalkanes [i.e., tetradecafluorohexane (TDFH), perfluoromethylcyclohexane, and so on], because of their low solubility. However, Curran et al. reported that the solubility of lightly fluorous compounds was improved by "solvent tuning", which involves increasing 
the polarity of fluorous solvents by the addition of polar fluorous solvents, such as hydrofluoroethers, to perfluoroalkanes [31,32]. In this method, when solvent tuning was performed by adding perfluoroalcohol, $1 H, 1 H, 2 H, 2 H$-tridecafluoro- $1-n$-octanol (TFO), to TDFH, extraction of ion-pair forms between the nucleotides and HFUA could be performed. Nucleotides in the fluorous solvent were back-extracted with a non-fluorous solution, such as ammonium hydroxide in acetonitrile, and subsequently analyzed by hydrophilic interaction liquid chromatography (HILIC) with UV detection (254 nm). In this method, the amide-type HILIC column was used for analyzing the nucleotides under alkaline mobile phase conditions. When a mixture of $200 \mathrm{mM}$ ammonium carbonate buffer ( $\mathrm{pH}$ 10.0), water, and acetonitrile was used as the mobile phase, the nucleotides could be moderately retained and separated with sharp peak shapes on the amide-type HILIC column.

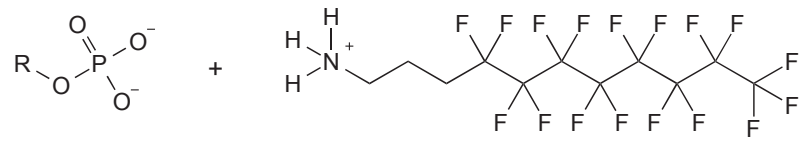

Nucleotides HFUA

Ion-pair formation Extraction to fluorous solvent

Fig. 7. Concept of fluorous ion-pair extraction for nucleotides utilizing HFUA.

In ion-pair extraction, the $\mathrm{pH}$ value of the sample also affects the extraction efficiency. In order to generate the ion-pair between the positively protonated amines of HFUA (predicted $\mathrm{pKa}$ value $=9.3$ ) and negatively deprotonated phosphates of the nucleotides, the $\mathrm{pH}$ of samples was set to 4.0 by adjusting with acetate buffer. Furthermore, to optimize the extraction conditions, the effect of HFUA concentration $(0-80 \mathrm{mM})$ was investigated. As the concentration of HFUA was increased, the extraction efficiency of monophosphate groups (AMP and GMP) was increased. However, those of di and triphosphate groups slightly decreased with increasing HFUA concentration. This may be because the ion-pair interaction becomes stronger with an increasing number of phosphates in a molecule and HFUA concentration. Therefore, $40 \mathrm{mM}$ HFUA was selected as the optimal concentration in this method. Under optimized conditions, the nucleotides could be effectively extracted with the simple liquid-liquid extraction system. In contrast, when the extraction of nucleotides was performed with a non-fluorous ion-pair reagent (n-octylamine) for comparison, they were not extracted to the fluorous solvent. We also confirmed that some structural analogues of the nucleotides (adenosine and cyclic AMP) could not be extracted with this method (less than 1\%). Accordingly, it was demonstrated that the combination of a fluorous ion-pair reagent and fluorous solvent enabled suitable and selective extraction of the nucleotides via fluorophilicity. A validation study was performed using standard solutions of the nucleotides. The correlation coefficients of the calibration curves in the range of $0.05-10 \mu \mathrm{M}$ were greater than 0.9998 . The LODs $(S / N=3)$ of the nucleotides in the present method were 9.7-24 nM. The extraction recovery obtained for spiked deionized water samples were between 70.4 and $96.8 \%$ with excellent reproducibility (Table 2 ).

Table 2. Extraction recovery of nucleotides.

\begin{tabular}{lccc}
\hline & \multicolumn{3}{c}{ Mean recovery $\pm \mathrm{SD}(\%, n=6)$} \\
\cline { 2 - 4 } & $0.5 \mu \mathrm{M}$ & $1.0 \mu \mathrm{M}$ & $10 \mu \mathrm{M}$ \\
\hline AMP & $74.4 \pm 3.2$ & $78.6 \pm 3.0$ & $75.7 \pm 2.0$ \\
ADP & $88.9 \pm 3.3$ & $85.0 \pm 1.6$ & $84.5 \pm 2.4$ \\
ATP & $70.4 \pm 2.6$ & $77.6 \pm 2.6$ & $75.9 \pm 2.3$ \\
GMP & $76.3 \pm 3.6$ & $77.4 \pm 2.9$ & $78.8 \pm 2.9$ \\
GDP & $96.8 \pm 2.1$ & $89.5 \pm 3.5$ & $90.3 \pm 2.1$ \\
GTP & $72.3 \pm 2.7$ & $75.3 \pm 4.7$ & $73.8 \pm 2.2$ \\
\hline
\end{tabular}

We applied this method to the determination of nucleotides in cell extract samples in order to investigate the utility for the selective extraction of nucleotides from biological samples. In this study, extracts from MCF-7 and MCF-10A cells, which are a human breast cancer cell line and normal cell line, respectively, were utilized (Fig. 8). The examined nucleotides in the cell extracts were successfully detected with this method, although some unidentified peaks, which might be phosphate-containing compounds, were also detected on the chromatograms. In contrast, in the case of analysis without extraction, some interference peaks ( $c$. 9 min), which might be non-phosphate-containing compounds derived from cell samples, were observed. These results indicate that the selectivity of this method was proved with the forward and back extraction behaviors of the cell samples.

\section{Conclusions}

Selective analysis methods for biogenic-related compounds were developed utilizing the fluorous derivatization and separation techniques. We firstly applied this technique to the analysis of native fluorescent biogenic amines. The fluorous derivatives of the amines were selectively retained on the fluorous LC column, whereas non-derivatized biological matrices were not. Therefore, the examined amines in human urine could be analyzed without any interference, and detected sensitively by the fluorescence detector. Furthermore, we applied the method to the determination of trace amounts of biogenic molecules by LC-MS/MS. In the case of analysis of biogenic primary amines, they were transformed to binary 
(A)

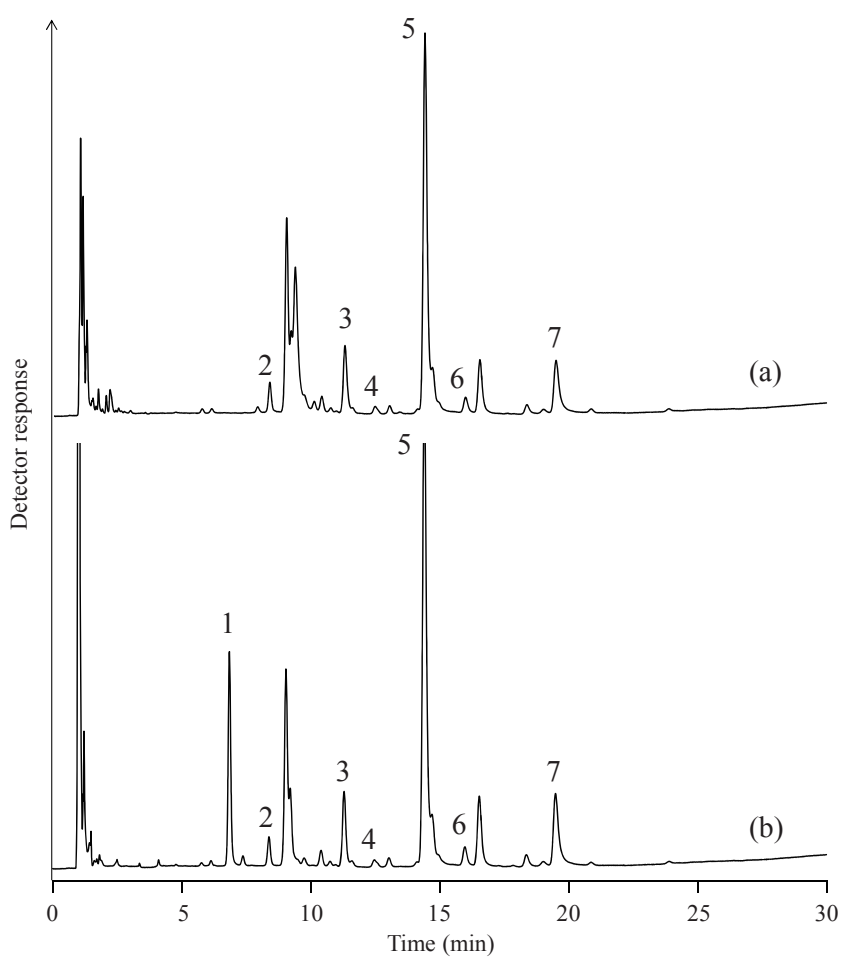

(B)

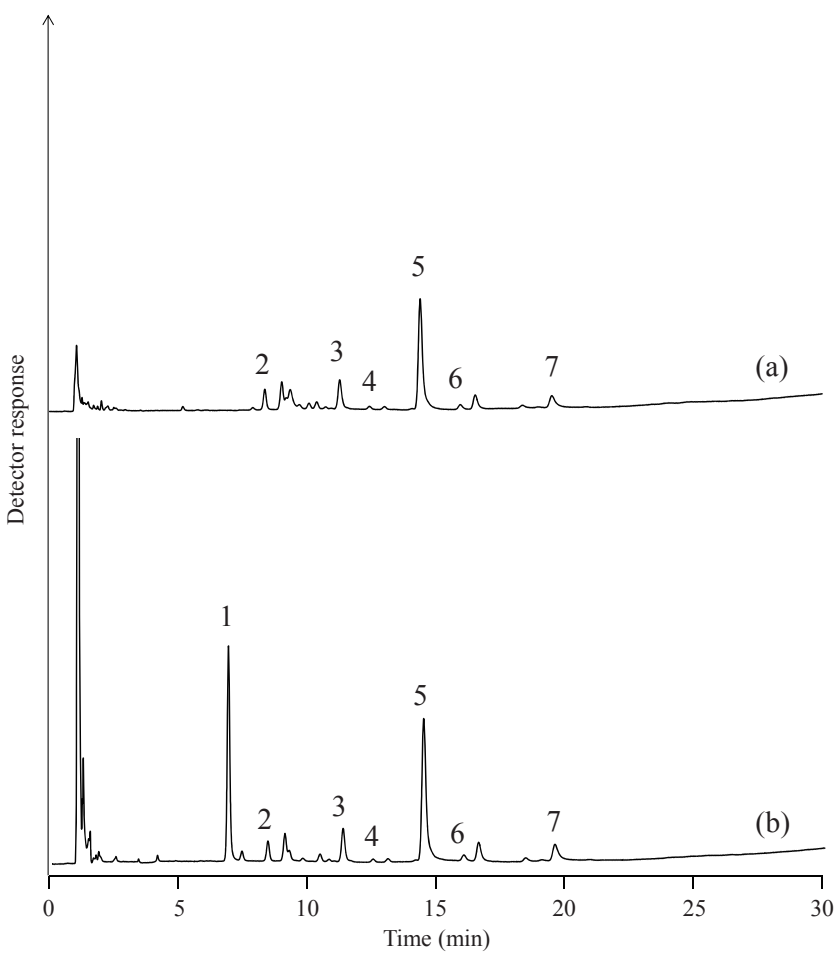

Fig. 8. Chromatograms obtained from (A) MCF-7 and (B) MCF-10A cell samples. (a) Non-extracted cell sample; (b) cell sample extracted with the present fluorous ion-pair extraction method. Peaks: 1; 6-Cl-PuDP (IS), 2; AMP, 3; ADP, 4; GMP, 5; ATP, 6; GDP, 7; GTP. Reprinted with permission from Ref. [30]. fluorous derivatives in order to utilize their strong fluorophilicity. The binary derivatives of amines were also highly retained on the fluorous LC column, and their endogenous levels in plasma could be determined accurately by MS/MS because matrix-induced effects were not observed. This method was also useful for the direct tandem MS analysis of amino acids. Amino acid derivatives were effectively purified with a monolithic F-SPE spin column, and therefore, they could be directly injected into the tandem MS system without LC column separations. This method was successfully applied to the diagnosis of amino acid metabolic abnormalities, such as MSUD and PKU. Moreover, the fluorous separation technique was utilized for the selective extraction of nucleotides without derivatization. The nucleotides were extracted with a fluorous ion-pair reagent into a fluorous solvent via a simple liquid-based extraction system. This extraction method enabled the analysis of nucleotides in cell extract samples.

We believe that fluorous affinity is a useful and powerful tool for a wide variety of biological analysis applications.

\section{Acknowledgement}

The author would like to express his gratitude to Professor Masatoshi Yamaguchi and Professor Hitoshi Nohta (Fukuoka University) for their supervision and valuable discussions on all work described here. The author is also grateful to Associate Professor Hideyuki Yoshida (Fukuoka University) and Associate Professor Kenichiro Todoroki (University of Shizuoka) for their important suggestions. The author gratefully acknowledges all collaborators and coworkers of his studies. This work was supported by JSPS KAKENHI, and by funding from the Central Research Institute of Fukuoka University.

Finally, the author is grateful to the Society for Chromatographic Sciences for selecting him as a recipient of the Encouragement Award in 2015 and giving him the opportunity to publish this focusing review.

\section{References}

[1] Gladysz, J. A.; Curran, D. P.; Horváth, I. T. Eds. Handbook of Fluorous Chemistry, Wiley-VCH: Weinheim, Germany, 2004.

[2] Uneyama, K. Organofluorine Chemistry, Blackwell Publishing: Oxford, U.K., 2006.

[3] Curran. D. P. Science 2008, 321, 1645-1646.

[4] Zhang, W. Chem. Rev. 2009, 109, 749-795.

[5] Todoroki, K.; Yoshida, H.; Hayama, T.; Itoyama, M.; Nohta, H.; Yamaguchi, M. J. Chromatogr. B 2011, 879, 1325-1337.

[6] Hayama, T.; Yoshida, H.; Yamaguchi, M.; Nohta, H. J. Pharm. Biomed. Anal. 2014, 101, 151-160. 
[7] Sakaguchi, Y.; Yoshida, H.; Hayama, T.; Itoyama, M.; Todoroki, K.; Yamaguchi, M.; Nohta, H. J. Chromatogr. A 2011, 1218, 5581-5586.

[8] Mashige, F.; Matsushima, Y.; Miyata, C.; Yamada, R.; Kanazawa, H.; Sakuma, I.; Takai, N.; Shinozuka, N.; Ohkubo, A.; Nakahara, K. Biomed. Chromatogr. 1995, 9, 221-225.

[9] Peterson, Z. D.; Collins, D. C.; Bowerbank, C. R.; Lee, M. L.; Graves, S. W. J. Chromatogr. B 2002, 776, 221-229.

[10] Yoshitake, M.; Nohta, H.; Yoshida, H.; Yoshitake, T.; Todoroki, K.; Yamaguchi, M. Anal. Chem. 2006, 78, 920-927.

[11] Annesley, T. M. Clin. Chem. 2003, 49, 1041-1044.

[12] Matuszewski, B. K.; Constanzer, M. L.; Chavez-Eng. C. M. Anal. Chem. 2003, 75, 3019-3030.

[13] Stokvis, E.; Rosing, H.; Beijnen, J. H. Rapid Commun. Mass Spectrom. 2005, 19, 401-407.

[14] Remane, D.; Wissebach, D. K.; Meyer, M. R.; Manner, H. H. Rapid Commun. Mass Spectrom. 2010, 24, 859-867.

[15] Hayama, T.; Sakaguchi, Y.; Yoshida, H.; Itoyama, M.; Todoroki, K.; Yamaguchi, M.; Nohta, H. Rapid Commun. Mass Spectrom. 2010, 24, 2868-2874.

[16] Sakaguchi, Y.; Hayama, T.; Yoshida, H.; Itoyama, M.; Todoroki, K.; Yamaguchi, M.; Nohta, H. Rapid Commun. Mass Spectrom. 2014, 28, 2481-2489.

[17] Hayama, T.; Sakaguchi, Y.; Yoshida, H.; Itoyama, M.; Todoroki, K.; Yamaguchi, M.; Nohta, H. Anal. Chem. 2012, 84, 8407-8414.

[18] Guo, K.; Ji, C.; Li, L. Anal. Chem. 2007, 79, 8631-8638.

[19] Ji, C.; Li, W.; Ren, X.; El-Kattan, A. F.; Kozak, R.; Fountain, S.; Lepsy, C. Anal. Chem. 2008, 80, 9195-9203.

[20] Zhang, W. J. Fluor. Chem. 2008, 129, 910-919.

[21] Xiao, N.; Yu, Y. B. J. Fluorine Chem. 2010, 131, 439-445.

[22] Cai, H. L.; Zhu, R. H.; Li, H. D. Anal. Biochem. 2010 , 396, 103-111.

[23] de John, W. H. A.; Graham, K. S.; van der Molen, J. C.; Links, T. P.; Morris, M. R.; Ross, H. A.; de Vries, E. G. E.; Kema, I. P. Clin. Chem. 2007, 53, 1684-1693.

[24] Mao, H. M.; Chen, B. G.; Qian, X. M.; Liu, Z. Microchem. J. 2009, 91, 176-180.

[25] Chace, D. H.; Millington, D. S.; Terada, N.; Kahler, S. G.; Roe, C. R.; Hofman, L. F. Clin. Chem. 1993, 39, 66-71.

[26] Nagy, K.; Takáts, Z.; Pollreisz, F.; Szabó, T; Vékey, K. Rapid. Commun. Mass Spectrom. 2003, 17, 983-990.

[27] Wang, C.; Zhang, W.; Song, F.; Liu, Z.; Liu, S. Amino Acids 2012, 42, 1889-1895.

[28] Tamashima, E.; Hayama, T.; Yoshida, H.; Imakyure,
O.; Yamaguchi, M.; Nohta, H. J. Pharm. Biomed. Anal. 2015, 115, 201-207.

[29] HMDB web site, http://www.hmdb.ca (accessed December 2015)

[30] Hayama, T.; Kiyokawa, E.; Yoshida, H.; Imakyure, O.; Yamaguchi, M.; Nohta, H. Chromatography 2015, 36, 13-18.

[31] Yu, M. S.; Curran, D. P.; Nagashima, T. Org. Lett. 2005, 7, 3677-3680.

[32] Chu, Q.; Yu, M. S.; Curran, D. P. Tetrahedron 2007, 63, 9890-9895. 